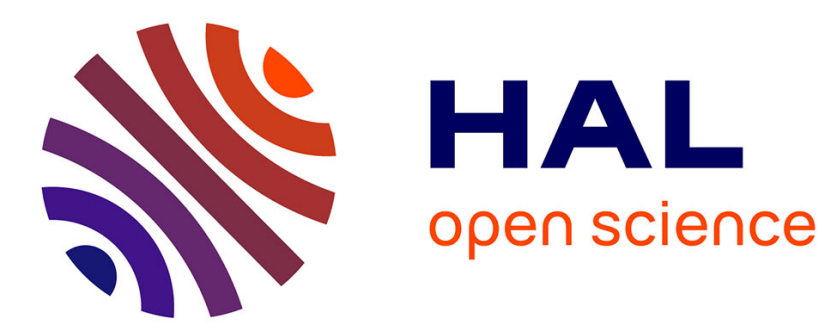

\title{
Promouvoir un management environnemental participatif
}

Angèle Renaud

\section{To cite this version:}

Angèle Renaud. Promouvoir un management environnemental participatif. Gestion - HEC Montréal, 2011, 36 (3), pp.80-89. hal-00908548

\section{HAL Id: hal-00908548 \\ https://hal.science/hal-00908548}

Submitted on 24 Nov 2013

HAL is a multi-disciplinary open access archive for the deposit and dissemination of scientific research documents, whether they are published or not. The documents may come from teaching and research institutions in France or abroad, or from public or private research centers.
L'archive ouverte pluridisciplinaire HAL, est destinée au dépôt et à la diffusion de documents scientifiques de niveau recherche, publiés ou non, émanant des établissements d'enseignement et de recherche français ou étrangers, des laboratoires publics ou privés. 


\title{
PROMOUVOIR UN MANAGEMENT ENVIRONNEMENTAL PARTICIPATIF
}

\author{
Angèle Renaud $^{1}$
}

Angèle Renaud est maitre de conférences en sciences de gestion à l'Université de Bourgogne, angele.renaud@u-bourgogne.fr 


\section{Résumé}

L'expérience nous montre que, faute de solliciter l'engagement des parties prenantes, il risque d'y avoir un écart important entre le discours des dirigeants et les pratiques réelles des entreprises en matière de management environnemental. Par ailleurs, les dirigeants doivent de plus en plus tenir compte des attentes de diverses parties prenantes en cette matière. Les clients, les employés, les fournisseurs et les communautés locales ne veulent plus seulement être informés ; ils tiennent aussi à participer à la prise de décisions environnementales étant donné qu'ils en subissent les conséquences. Dans ce contexte, la question se pose: qui faire participer et comment? Cet article vise à aider les dirigeants qui veulent amorcer une démarche de management environnemental participatif. Pour y parvenir, nous montrons comment les parties prenantes internes et externes s'engagent au sein de 10 entreprises jugées proactives en terme de management environnemental. Puis, nous décrivons les processus de participation vertical, transversal et externe. Nous faisons également trois recommandations pour aider les dirigeants à mettre en place un management environnemental participatif : instaurer une communication régulière parmi le personnel de tous les niveaux et de tous les secteurs, structurer ou institutionnaliser la préoccupation environnementale en mandatant des responsables et encourager la participation des parties prenantes externes.

Mots clés : participation, parties prenantes, management environnemental, performance environnementale, norme ISO 14001, entreprises proactives, responsabilité sociale de l'entreprise (RSE)

Fonctions : management, management international, gestion des opérations et de la production (GOP) 


\title{
Promouvoir un management environnemental participatif
}

\begin{abstract}
Angèle Renaud
Aujourd'hui, les entreprises doivent répondre des conséquences sociales et environnementales de leurs activités économiques. Face aux interpellations de la société, la «responsabilité sociale de l'entreprise » (RSE) se traduit par l'intégration d'objectifs sociaux et environnementaux dans la stratégie et la prise en compte des attentes des parties prenantes. Au nom de cette responsabilité, les dirigeants d'entreprises adoptent des instruments permettant d'opérationnaliser les objectifs stratégiques annoncés et de les intégrer aux systèmes d'information et de pilotage ${ }^{2}$. Parmi ces instruments, on parle de plus en plus du management environnemental comme d'un «outil de gestion permettant aux entreprises de contrôler et réduire les impacts environnementaux de leurs opérations ${ }^{3} »$, et ce, surtout depuis la publication de la norme ISO $14001^{4}$. À ce jour, plus de 223149 certificats ISO 14001 ont été délivrés dans 159 pays 5 .
\end{abstract}

Soulignons que le management environnemental ${ }^{6}$ ne se réduit pas à l'adoption de la norme ISO 14001, cette dernière ne constituant qu'un pas vers un véritable engagement écologique des entreprises. Depuis quelques années, plusieurs études canadiennes, états-uniennes et françaises ${ }^{7}$ mettent d'ailleurs l'accent sur les limites et les effets pervers potentiels de cette certification. En outre, lorsque la quête de la «légitimité sociale $^{8} \gg$ constitue la principale raison d'obtenir la norme ISO 14001, il y a souvent un décalage entre les pratiques réelles de management environnemental et les exigences de cette norme. Pour reprendre les propos de Boiral (2007a, 2007b), «le management environnemental aurait donc deux côtés pas toujours cohérents : le "côté jardin" prenant en compte les apparences, les discours des dirigeants, les politiques officielles, les engagements institutionnels, etc.; et le "côté cour" beaucoup moins apparent, celui des pratiques internes, des comportements de travail, des préoccupations quotidiennes pour l'environnement, des mesures concrètes de prévention de la pollution, etc.».

Gestion, Revue internationale de gestion - Vol. $36 N^{\circ} 3,2011-$ A. RENAUD 
Un tel écart entre le discours et la pratique peut s'expliquer par le manque d'engagement du personnel dans la démarche environnementale. En effet, la norme ISO 14001 ne comporte aucune recommandation visant à adopter une démarche participative ni à promouvoir un climat de confiance, de mobilisation ou d'écoute du personnel pour y parvenir' ${ }^{9}$. Bien que certains articles de cette norme présentent des directives en matière de communication interne, celles-ci restent peu nombreuses, vagues et peu respectées ${ }^{10}$. Comme la norme insiste surtout sur les mesures ou les contrôles, les études indiquent que la majorité des employés et des cadres intermédiaires appartenant à des organisations certifiées ISO 14001 n'adhèrent pas réellement à ce type de norme ou le font de façon rituelle pour satisfaire en surface aux exigences de l'audit de certification ${ }^{11}$. En matière de communication externe, les exigences de cette norme sont plutôt minces et se résument à «mettre en œuvre et tenir à jour des procédures pour recevoir et documenter les demandes des parties prenantes externes et y apporter les réponses correspondantes» (art. 4.4.3). Autrement dit, comme le déclare Gendron (2004 : 217), «une entreprise peut se contenter de recueillir les plaintes des parties intéressées et les réponses correspondantes dans un cahier spécialement consacré à cette fin en établissant une procédure à cet effet».

Or, le management environnemental étant un domaine à responsabilité partagée qui a des incidences sur nombre d'acteurs, plusieurs auteurs recommandent de faire participer l'ensemble des parties prenantes ${ }^{12}-$ les employés, les clients, les fournisseurs, les collectivités territoriales, etc. - afin de mettre en œuvre les moyens les plus adaptés et optimaux possible à leurs attentes respectives ${ }^{13}$. Le management environnemental suppose avant tout une approche participative et une large mobilisation des employés. Cette participation serait indispensable, car l'implantation des décisions environnementales dans les opérations quotidiennes implique qu'elles soient intégrées et appliquées par l'ensemble du personnel ${ }^{14}$. D'ailleurs, et comme l'affirme Gendron (2004 : 126), «c'est souvent au niveau opérationnel, en changeant de simples pratiques, que l'on peut obtenir une amélioration rapide de la performance environnementale, et ce à faible coût. Par la suite, les suggestions des employés permettront d'identifier des occasions intéressantes et, en les consultant, on pourra éviter des investissements inappropriés.»

Gestion, Revue internationale de gestion - Vol. $36 N^{\circ}$ 3, $2011-A$. RENAUD 
Comme le notent Sobczak et Berthoin-Antal (2010 : 116), les dirigeants ont de moins en moins la liberté de décider sans solliciter la participation des parties prenantes en matière de management environnemental : «[...] ces dernières ne se contentent plus uniquement d'évaluer les performances réalisées par les entreprises dans différents domaines [économiques, sociaux et environnementaux] et de leur demander des comptes sur le passé. [Elles] cherchent à influencer le processus même de décision dans les entreprises afin que celui-ci intègre leurs intérêts en amont, dès qu'il s'agit de définir les objectifs futurs.». De fait, les diverses parties prenantes ne veulent plus se contenter d'être informées a posteriori des performances environnementales; «elles croient au contraire que leur participation au processus décisionnel est légitime dans la mesure où ce sont elles qui en subissent les conséquences ${ }^{15} \gg$. D'ailleurs, plusieurs entreprises ont fait évoluer leur management réactif, qui se réduisait au respect des normes en matière d'enjeux sociaux et environnementaux, vers un management proactif et interactif, qui se traduit par un engagement de leurs parties prenantes, ce qui permet de traiter efficacement ces enjeux ${ }^{16}$.

L'objectif de cet article est d'aider les dirigeants qui veulent entreprendre une démarche de management environnemental participatif en montrant comment les parties prenantes participent au management environnemental au sein d'entreprises jugées proactives en la matière. Pour ce faire, notre réflexion s'articule en deux temps. Dans un premier temps, nous illustrons le rôle des parties prenantes au sein de 10 entreprises proactives en matière de management environnemental (voir l'encadré 1). Dans un second temps, les enseignements tirés de ces cas nous permettent de formuler des recommandations pour les dirigeants souhaitant mettre en place un management environnemental dans lequel les parties prenantes puissent s'engager réellement.

Gestion, Revue internationale de gestion - Vol. $36 N^{\circ} 3,2011-A$. RENAUD 


\section{Encadré 1 - À propos de l'étude}

L'étude de cas repose sur l'analyse des trois derniers rapports sur le développement durable des 10 entreprises françaises certifiées ISO 14001 retenues pour cette recherche et sur la conduite de 30 entretiens individuels réalisés auprès de hauts dirigeants, dirigeants opérationnels et responsables en matière d'environnement ou de développement durable travaillant pour ces entreprises. Les entrevues cherchaient à comprendre les pratiques de management environnemental et à étudier le rôle accordé aux parties prenantes dans ces entreprises. Pour mettre en évidence différentes expériences en cette matière, nous avons sélectionné des PME et de grandes entreprises appartenant à divers secteurs d'activité : aéronautique, commerce et distribution, chimie, gaz industriels, plasturgie, services et agroalimentaire. Ces entreprises ont été choisies pour leur attitude jugée proactive en matière de stratégies vertes car, conformément à la littérature ${ }^{17}$, elles vont au-delà de la conformité aux lois et aux réglementations environnementales, font intervenir la dimension écologique dans leurs stratégies et intègrent diverses parties prenantes à leur processus décisionnel.

L'engagement des parties prenantes en matière de management environnemental : qui et comment?

$\mathrm{Au}$ sein des organisations proactives, le management environnemental est une préoccupation partagée et non le fait de certains acteurs, tels que les responsables de l'environnement ou du développement durable. Les parties prenantes engagées dans le processus de management environnemental peuvent être regroupées en deux catégories : les parties prenantes internes, comme les dirigeants, les responsables de l'environnement ou du développement durable ainsi que les employés, et les parties prenantes externes, comme les clients et les consommateurs, les fournisseurs et les autorités publiques ${ }^{18}$.

Gestion, Revue internationale de gestion - Vol. $36 N^{\circ} 3,2011-A . R E N A U D$ 


\section{L'engagement des parties prenantes internes}

Les dirigeants. D'après la norme ISO 14001, l'engagement des dirigeants constitue une condition sine qua non au succès du management environnemental. Cet engagement se traduit par la définition d'une politique environnementale, l'allocation des ressources aux responsables de l'environnement et la place et l'importance de la fonction «environnement» dans l'organisation ${ }^{19}$. La norme exige également la planification d'une réunion à intervalles réguliers, appelée «revue de direction», nécessitant la participation des hauts dirigeants de l'organisation. La revue de direction permet de discuter des préoccupations écologiques, de débattre des performances environnementales de l'entreprise et de prendre des décisions stratégiques afin d'améliorer ces performances. Au sein des organisations proactives étudiées, on constate un engagement régulier des dirigeants de différents niveaux hiérarchiques et de divers secteurs. En effet, le management environnemental apparaît comme une question qui mobilise différentes fonctions de l'entreprise : les services de l'environnement, de la production, de la recherche, de la communication, des ressources humaines, des achats, des finances, du marketing, etc.

«Au niveau national, des revues de direction sont organisées deux fois par an dans le cadre de la norme ISO 14001 avec le directeur de l'entreprise, tous les directeurs des régions, le directeur QSE [qualité, sécurité et environnement], les relais QSE des régions; autour de la table, tout le monde parle des incidents, etc. À mon niveau, qui est le niveau régional, un point QSE est effectué au cours de réunions mensuelles avec tous les chefs de services et le responsable commercial. On fait un suivi de ce qui ne va pas, du choix des produits non nocifs, on aborde aussi l'aspect environnemental à travers les audits qui ont été réalisés.» (un directeur régional dans une entreprise chimique)

«Il y a des revues de direction environnementale qui sont des grands-messes réalisées avec l'ensemble du comité de direction, et qui ont lieu tous les six mois en général. Les personnes qui participent à ces revues de direction sont le comité de direction, en plus de l'équipe QSE et de quelques directeurs opérationnels. Ces réunions comportent une partie opérationnelle, qui permet de revoir les indicateurs et de définir des orientations visant à améliorer ces indicateurs, et une partie stratégique, où des orientations stratégiques sont définies pour tous les grands projets d'investissement; ces projets sont systématiquement étudiés, ce

Gestion, Revue internationale de gestion - Vol. $36 N^{\circ} 3$, 2011-A. RENAUD 
qui permet de prendre en compte leurs impacts environnementaux.» (un directeur opérationnel dans une entreprise agroalimentaire)

Par ailleurs, les entreprises étudiées vont au-delà des recommandations de la norme ISO 14001, étant donné que la participation des dirigeants au management environnemental ne se limite pas aux revues de direction. En dehors de ces réunions, les questions environnementales sont examinées régulièrement dans les comités exécutifs, les comités de direction et les comités régionaux organisés mensuellement par les hauts dirigeants, auxquels se joignent les principaux dirigeants de l'entreprise.

«On évoque l'environnement également dans nos réunions mensuelles avec nos régions. Dans les comités de direction aussi, on en discute chaque fois, de façon très rigoureuse.» (le directeur général d'une entreprise chimique)

Ces comités sont décrits par les personnes interviewées comme des lieux de discussion favorisant l'interaction et la résolution collective des problèmes. Au cours de ces rencontres, le tableau de bord vert constitue l'outil autour duquel s'organisent les débats. Cet outil regroupe des indicateurs clés servant à mesurer et à piloter la performance environnementale de l'entreprise. Il permet ainsi d'attirer l'attention des dirigeants sur les priorités stratégiques et de réagir aux dérives mises en évidence.

«Nous avons un comité de pilotage mensuel avec le comité de direction. Au cours de cette réunion, nous présentons les indicateurs et les points de repère, puis nous débattons d'aspects devant être améliorés. Il s'agit d'un processus d'amélioration continue et de décision collégiale.» (un directeur de l'environnement dans une entreprise du secteur gazier)

«Au niveau de l'entreprise, des objectifs de groupe sont fixés et un certain nombre d'indicateurs sont mis en place. Ces indicateurs permettent de vérifier, par exemple, si les objectifs d'économie d'énergie, de consommation d'eau, de traitement des déchets traités dans le cadre des plans d'action de la certification ISO 14001 sont respectés. Tous ces indicateurs sont alors inclus dans le tableau de bord de l'entreprise et étudiés par le PDG et les autres dirigeants dans le contexte d'un comité exécutif.» (un responsable de l'environnement dans une entreprise du secteur aéronautique)

Gestion, Revue internationale de gestion - Vol. $36 N^{\circ} 3,2011-A$. RENAUD 
Les responsables de l'environnement. Selon la taille des entreprises étudiées, les services environnementaux sont plus ou moins décentralisés afin de permettre aux professionnels de l'environnement de discuter fréquemment avec les dirigeants et les employés, et cela à différents niveaux de l'organisation (les divisions, les fonctions, les métiers), en vue de mobiliser tous et chacun dans la démarche environnementale. Dans les grandes entreprises, ces experts en environnement sont regroupés en réseau (appelé «réseau vert» ou «réseau environnement») avec à sa tête un directeur de l'environnement qui est appuyé dans ses missions par une équipe d'experts et un responsable de l'environnement dans chaque unité opérationnelle (comme les usines ou les magasins) ${ }^{20}$.

Le directeur de l'environnement ou du développement durable participe à l'élaboration de la stratégie verte avec la direction générale et veille à la traduire dans des actions concrètes au sein de l'entreprise. Il est également chargé du pilotage de la performance environnementale et de la publication du rapport sur le développement durable. Il fait partie du comité de l'environnement qui se réunit chaque année en une revue de direction pour fixer les objectifs, négocier le budget nécessaire à la mise en place des actions et décider des améliorations à apporter au système de management environnemental et à la stratégie verte.

«Ma mission est de définir la politique environnementale du groupe avec la direction générale pour améliorer la performance environnementale du groupe, de faciliter sa mise en œuvre en favorisant les échanges entre les différents sites industriels, de garantir la qualité et la pérennité du système de management environnemental à travers les auditeurs qualifiés et d'animer tout le réseau d'environnement du groupe.» (un directeur de l'environnement dans un groupe de plasturgie)

«Je reçois des informations du terrain sous forme de tableau de bord. Moi-même, j'ai un rapport à faire au groupe, je centralise toutes les données environnementales qui donnent lieu à un rapport sur le développement durable pour l'ensemble du groupe.» (un directeur de l'environnement dans une entreprise du secteur gazier)

Gestion, Revue internationale de gestion - Vol. $36 N^{\circ} 3,2011-A$. RENAUD 
$\mathrm{Au}$ niveau local, les responsables de l'environnement (parfois appelés «correspondants environnementaux» ou «animateurs environnementaux») réalisent les analyses environnementales, suivent la mise en œuvre des programmes environnementaux, préparent le rapport environnemental, planifient les audits environnementaux internes et communiquent avec les employés et les dirigeants locaux. Ils veillent aussi à l'application de la réglementation en relation avec les autorités locales et organisent la formation et la sensibilisation du personnel. Puis, ils participent aux revues de direction locales avec les responsables des emplacements opérationnels. Lorsqu'ils constatent un écart majeur pendant le pilotage des performances environnementales, ils informent les dirigeants locaux et le directeur de l'environnement de l'entreprise. En effet, les responsables de l'environnement sont rattachés à la direction locale et représentent les interlocuteurs privilégiés du directeur de l'environnement dans les emplacements. À ce titre, ils jouent un rôle clé dans l'articulation de la stratégie verte de l'entreprise.

\begin{abstract}
«Les correspondants environnementaux pilotent les activités au quotidien dans le domaine environnemental. Chaque correspondant, pour sa zone, est chargé de procédures de gestion de l'environnement, de prévention en matière d'environnement; il applique au niveau de son secteur les directives du manuel sur l'environnement que nous avons dans l'établissement. Une fois par an, il participe à la revue de direction globale où l'on relève ce qui fonctionne bien, ce qui fonctionne moins bien, et des axes de progrès pour les années futures.» (un directeur d'usine dans un groupe de plasturgie)
\end{abstract}

À travers le réseau vert, les responsables de l'environnement des différents emplacements discutent fréquemment. Ils organisent des réunions pour traiter des problèmes, parler de l'évolution de la législation, partager les bonnes pratiques environnementales dans l'ensemble des emplacements et réfléchir à des initiatives susceptibles d'améliorer la stratégie environnementale de l'entreprise. Ces responsables sont assistés par une équipe d'experts, des spécialistes du droit de l'environnement, des ingénieurs chargés de résoudre les problèmes spécifiques (pollution de l'air, de l'eau, du sol, nuisances sonores, déchets dangereux, etc.).

Gestion, Revue internationale de gestion - Vol. $36 N^{\circ} 3,2011-A$. RENAUD 
«Il y a un réseau de responsables d'énergie qui se réunit régulièrement, qui échange les bonnes pratiques, qui fait le point sur les résultats, etc. Ce réseau crée des relations entre les différentes entités pour que toutes avancent de façon homogène.» (un directeur opérationnel dans une entreprise du secteur aéronautique)

«On a un réseau assez important en environnement puisqu'on possède un laboratoire engagé dans les systèmes de management environnemental. Ce laboratoire comprend un responsable de l'air, un responsable de l'eau, etc., qui participent activement au suivi des indicateurs et de la performance environnementale selon ces différentes thématiques.» (un responsable de l'environnement dans une entreprise du secteur aéronautique)

Les employés. Toutes les personnes rencontrées sont d'avis que l'efficacité du management environnemental repose essentiellement sur la mobilisation des employés. Sans l'adhésion de ces derniers, le management environnemental semble voué à l'échec. Ainsi, pour être vraiment efficaces, les employés sont consultés régulièrement aux différentes étapes du système de management environnemental : la planification, la mise en œuvre, le contrôle et l'évaluation des résultats. Au cours de la phase de planification, les employés participent aux analyses environnementales pour repérer et évaluer les impacts environnementaux de leurs activités. Ces impacts servent ensuite à fixer les objectifs du programme environnemental de l'entreprise. Ainsi, les employés participent dès le démarrage de la démarche environnementale.

«La première étape est l'analyse complète de nos activités pour déterminer leurs impacts sur le sol, le soussol, l'air, l'eau, le bruit, etc., de manière à mettre en place des plans d'action sur ces aspects. Le personnel a été associé dès le début parce qu'il faut voir avec les gens du terrain les impacts des activités sur l'environnement. Et si les employés n'adhèrent pas, on ne peut pas avancer...» (un expert en environnement dans une entreprise du secteur gazier)

Puis, pendant la phase de mise en œuvre du système, l'ensemble du personnel est formé et sensibilisé sur une base régulière. Ces formations, qui ont pour but de mobiliser durablement les employés autour des objectifs environnementaux, portent sur des thématiques générales (exigences réglementaires ou de la 
norme ISO 14001, stratégie environnementale, etc.) et particulières (impacts environnementaux, actions à réaliser, responsabilités, cibles de performance, rapports et audits internes, gestion des produits chimiques et des déchets dangereux, etc.).

«On a une batterie de formations sur la norme ISO 14001, mais aussi sur d'autres thèmes tels que l'énergie, la haute qualité environnementale, les produits chimiques, les transports de matières dangereuses ou encore l'amiante. Des formations sont assurées aussi bien sur le rôle de correspondant de l'environnement que sur les audits internes.» (un responsable de l'environnement dans une entreprise du secteur aéronautique)

Pour amener les employés à modifier leurs comportements au quotidien, les dirigeants n'hésitent pas à leur confier certaines tâches environnementales. De plus, les employés sont fréquemment consultés et associés à la définition des actions en matière d'environnement. Ces consultations peuvent prendre différentes formes : groupes de travail, réunions d'information, mise en place de boîtes à idées, utilisation de l'intranet, échanges informels, etc. Selon les entretiens réalisés, il semble que ces consultations favorisent la prise en compte des savoirs tacites des employés, nourrissent l'apprentissage organisationnel et contribuent à l'amélioration des performances environnementales.

«On a créé un "groupe de gestes verts" composé d'assistantes et de secrétaires qui construisent un plan annuel de communication, de campagne sur la consommation d'électricité, sur l'utilisation de papier recto verso, bref, sur tout ce qui est lié aux activités tertiaires et aux bureaux. On a mis sur pied des groupes de travail participatif de 8 ou 10 personnes de tous les niveaux hiérarchiques. Chaque groupe de travail devait établir la cartographie de ses processus. On a élaboré ainsi trois plans d'action avec les services : un plan d'action pour les sites opérationnels, qui gère les impacts environnementaux des lieux de production; un plan d'action dans les vignobles; un plan d'action pour les produits, qui se penche sur les impacts des produits qu'on met sur le marché ou sur des actions plus transversales.» (un directeur de l'environnement dans une entreprise agroalimentaire)

«Tous les trimestres, nous faisons des réunions de service où sont abordés des thèmes relatifs à la qualité, à la sécurité et à l'environnement. Nous avons également un système de communication dans lequel les directeurs opérationnels peuvent soumettre des suggestions, des informations et des idées. Nous passons

Gestion, Revue internationale de gestion - Vol. $36 N^{\circ} 3$, 2011-A. RENAUD 
tous les jours dans les ateliers pour discuter de manière informelle avec les gens. C'est également à nous d'aller sur le terrain pour faire participer les employés et les motiver régulièrement au lieu de rester dans nos bureaux.» (un expert en environnement dans une entreprise du secteur gazier)

Pour faire participer davantage les employés à la démarche environnementale, certaines entreprises ont instauré un régime de rémunération variable visant à souligner l'atteinte de résultats en cette matière. Mais pour l'heure, cette pratique reste limitée.

«Dans les accords d'intéressement qu'on a mis en place pour notre personnel, des éléments de développement durable sont intégrés, dont le niveau de consommation électrique et le niveau de consommation d'eau constituent deux indicateurs.» (le PDG d'une entreprise agroalimentaire)

Enfin, les employés participent à l'évaluation des performances environnementales de leur entreprise en effectuant périodiquement des audits internes avec le service de l'environnement. Selon la norme ISO 14001, les auditeurs internes doivent effectuer ces vérifications avec compétence et impartialité. Pour respecter ces exigences, les entreprises que nous avons étudiées organisent pour leurs auditeurs internes des formations qui sont données par des cabinets d'audits externes. Ensuite, afin de démontrer leur indépendance, les auditeurs internes réalisent des audits croisés, c'est-à-dire qu'ils vont auditer une activité dans laquelle ils n'ont aucune responsabilité. Les audits croisés permettent de vérifier la conformité des pratiques environnementales aux réglementations, aux exigences de la norme ISO 14001, aux engagements formulés dans la politique environnementale, aux objectifs environnementaux, et éventuellement d'apporter des actions correctives.

«On est audité tous les ans à l'interne pour s'améliorer en permanence. Les audits sont réalisés par une équipe qui est formée et qui comprend aussi bien des membres du siège social que des gens du magasin, en plus du service du développement durable qui va d'un magasin à l'autre.» (un directeur de magasin dans une entreprise de commerce et de distribution)

Gestion, Revue internationale de gestion - Vol. $36 N^{\circ}$ 3, $2011-A$. RENAUD 
«On a un système d'audit croisé qui consiste, par exemple, pour les auditeurs de l'aéroport A, qui ont des connaissances techniques poussées dans la gestion de l'eau, à aller auditer les autres aéroports. Nous, on prend les audits de façon très positive dans la mesure où s'appliquent les nouvelles orientations et les axes d'amélioration et de progrès.» (un responsable de l'environnement dans une entreprise du secteur aéronautique)

En résumé, les employés participent aux différentes phases du management environnemental, ce qui leur permet d'acquérir de nouvelles connaissances grâce à la formation, à l'expérience et à l'échange de bonnes pratiques. Cette participation favorise l'apprentissage et s'inscrit dans une dynamique d'amélioration continue.

\section{L'engagement des parties prenantes externes}

Selon les entreprises proactives étudiées, la communication environnementale tient une place fondamentale dans leur stratégie. Cette communication ne se limite pas à la publication des rapports sur le développement durable, à des lettres d'information adressées aux parties prenantes ou à la mise en place d'un site Internet dédié aux impacts environnementaux des entreprises. Allant au-delà de la diffusion d'information, elle intègre une collaboration soutenue des parties prenantes externes au processus de décision. En effet, d'après les dirigeants rencontrés, ils organisent régulièrement des rencontres avec des parties prenantes externes (riverains, clients, fournisseurs, collectivités territoriales, etc.) pour discuter de leur stratégie et de leurs performances environnementales. Ce dialogue apparaît comme un mécanisme «gagnant-gagnant», car, d'une part, il contribue à légitimer les activités économiques des entreprises visà-vis de la société et à construire leur avantage concurrentiel et, d'autre part, il permet aux parties prenantes d'exprimer leurs attentes et d'intervenir dans le processus de décision pour s'assurer que cellesci seront prises en compte, notamment au moment de la définition des objectifs et des plans d'action.

«On se fixe des objectifs environnementaux, sur un horizon de deux ou trois ans, qui vont intéresser nos parties prenantes, c'est-à-dire l'entreprise et les riverains. On a des contacts réguliers avec les riverains. En

Gestion, Revue internationale de gestion - Vol. $36 N^{\circ} 3,2011-A . R E N A U D$ 
outre, on prépare chaque année un plan d'action pour se rapprocher petit à petit des objectifs qu'on s'est donnés. Aujourd'hui, on anticipe beaucoup, ce qui nous permet de limiter les impacts qu'on occasionne aux riverains ou au milieu naturel.» (un directeur d'établissement dans une entreprise chimique)

Il reste à savoir comment ces parties prenantes interviennent dans le processus de décision. En réalité, elles s'engagent de différentes façons : elles sont conviées à des réunions d'information ou de travail sur certaines problématiques; elles disposent de lieux de rencontres dédiés aux questions écologiques; elles participent aux journées portes ouvertes, aux visites d'entreprises et aux conférences-débats organisées par les entreprises; elles profitent aussi d'échanges informels pour véhiculer leurs messages; etc.

«Sur le plan des relations territoriales, nous avons beaucoup d'échanges concernant les effets de nos activités, avec la mise en place d'une maison d'accueil ${ }^{21}$ pour tous les riverains, dans laquelle les personnes qui le souhaitent peuvent prendre connaissance du fonctionnement de l'aéroport, de l'impact de nos activités au point de vue sonore.» (un responsable de l'environnement dans une entreprise aéronautique)

«Mon métier est de veiller à ce que tout ce qui se passe en France se fasse de manière cohérente en contact avec les parties prenantes, notamment les pouvoirs publics et les syndicats. Il m'est arrivé aussi de participer à une réunion avec un fournisseur qui a investi chez nous ou d'assister à une inauguration en compagnie du maire.» (le directeur général d'une entreprise chimique)

«L'entreprise sensibilise les clients en leur donnant des conseils au quotidien, en organisant des débats sur les grands enjeux environnementaux et en multipliant les occasions de rencontres entre les experts et le grand public; par exemple, chaque trimestre, des conférences sont tenues avec des scientifiques et des explorateurs.» (extrait du rapport sur le développement durable d'une entreprise de commerce et de distribution)

Soulignons que ce ne sont pas toutes les parties prenantes externes qui sont consultées. A contrario, celles qui participent aux décisions ne sont pas forcément les mêmes d'une entreprise à l'autre. Dans certaines entreprises étudiées, l'attention des dirigeants est davantage portée sur les riverains, tandis que dans d'autres entreprises, ce sont les clients, les fournisseurs ou les autorités locales qui sont visés. L'intérêt des

Gestion, Revue internationale de gestion - Vol. $36 N^{\circ} 3,2011-A . R E N A U D$ 
dirigeants pour certaines parties prenantes pourrait s'expliquer par l'un des facteurs mis en évidence par Mitchell et al. (1997) : l'urgence (l'état d'urgence des demandes), le pouvoir (la capacité d'influer sur les décisions de l'entreprise) et le degré de légitimité (la reconnaissance sociale).

\section{Comment adopter un management environnemental participatif?}

Afin d'améliorer l'efficacité de leur management environnemental, les entreprises peuvent solliciter la participation de leurs parties prenantes à leur prise de décision dans le but d'aboutir à un consensus sur la stratégie et la performance environnementales des entreprises. Pour implanter ce type de gestion, nous recommandons aux dirigeants d'instaurer un processus continu de consultation, de communication et de dialogue avec les différentes catégories de parties prenantes. Au regard des résultats de l'étude de cas, et comme l'illustre le schéma 1, ce processus peut prendre trois formes: verticale, transversale et externe. L'efficacité de ces différentes formes dépend de l'engagement réel des parties prenantes, de la transparence des informations communiquées, de l'écoute et du respect mutuel. Plus précisément, pour implanter ces processus de participation, nous proposons divers moyens de réaliser les trois préalables clés suivants : instaurer une communication régulière parmi le personnel de tous les niveaux et de tous les secteurs, structurer ou institutionnaliser la préoccupation pour l'environnement en mandatant des responsables et encourager la participation des parties prenantes externes.

Gestion, Revue internationale de gestion - Vol. $36 N^{\circ} 3,2011-A . R E N A U D$ 


\section{Schéma 1 - Les trois formes de participation des parties prenantes au management environnemental}

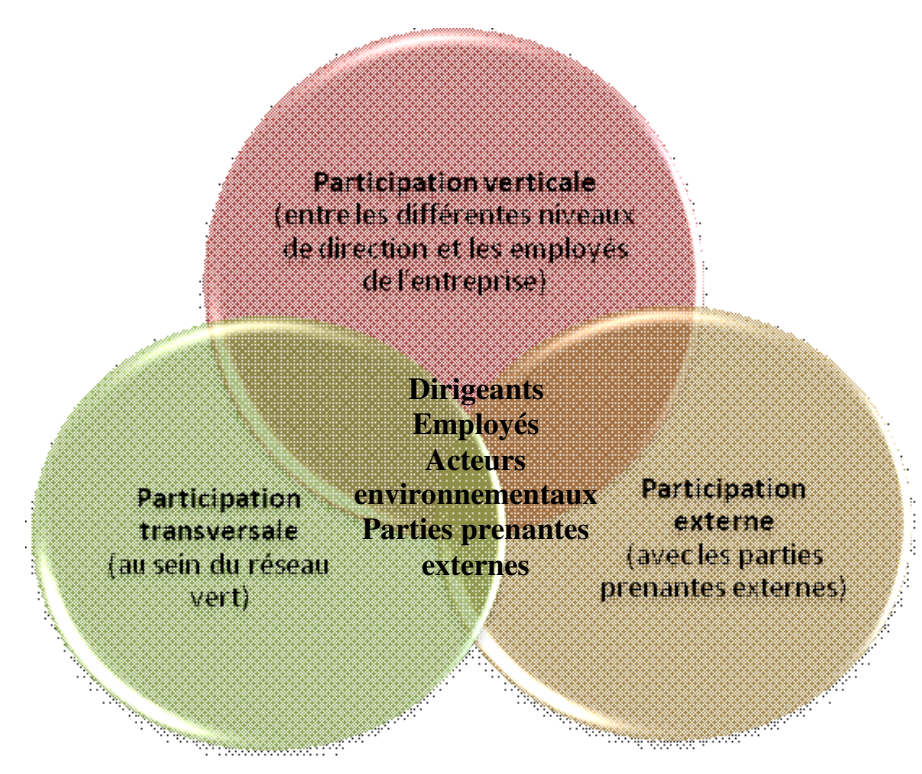

Instaurer une communication régulière parmi le personnel de tous les niveaux et de tous les secteurs

L'efficacité du management environnemental repose sur l'échange des savoirs entre les différents acteurs de l'entreprise. Pour favoriser une bonne circulation des informations environnementales, il faut multiplier les occasions de rencontre et de dialogue entre les dirigeants et leurs employés. En matière de communication verticale, la revue de direction constitue l'une des exigences de la norme ISO 14001. Cette réunion, planifiée à intervalles réguliers, a pour objectif d'amener les dirigeants à évaluer périodiquement leur système de management environnemental. Mais les débats ne doivent pas se limiter à cette revue de direction, comme nous l'avons vu dans le cas des entreprises étudiées; des réunions en face-à-face doivent être organisées où l'on discutera de problématiques environnementales (groupes de travail, réunions de service, réunions post-audit, débats en comité de direction, etc.).

Gestion, Revue internationale de gestion - Vol. $36 N^{\circ} 3,2011-A$. RENAUD 
Pour amener les acteurs à participer davantage et de manière régulière, il est opportun d'établir d'autres moyens de communication plus conviviaux. Il peut s'agir, par exemple, d'un forum de discussion pour permettre aux employés de s'exprimer librement et de transmettre des idées ou des suggestions aux dirigeants sur les pratiques de l'entreprise. Les dirigeants opérationnels ou les responsables de l'environnement pourront organiser et animer des débats dans le forum une fois par mois sur une thématique environnementale. Contrairement aux boîtes à idées, les forums de discussion sont interactifs et conviviaux ; ils constituent une occasion de créer un lien social et de fédérer les acteurs autour d'un même but.

De plus, les bonnes pratiques ainsi que les performances environnementales d'un emplacement en particulier pourront être régulièrement affichées dans tous les locaux de l'entreprise (tableaux d'affichage, bornes interactives, écrans d'information, etc.). Il s'agit également d'une manière de récompenser ces acteurs et d'inciter les autres à les imiter. Des dirigeants peuvent aller plus loin et récompenser financièrement les meilleures idées afin d'encourager la participation des employés.

D'autres opérations ponctuelles, telles que des visites du directeur général sur les lieux de production ou de commercialisation, des petits-déjeuners-débats et des conférences-débats avec les employés où ces derniers pourraient véritablement questionner les dirigeants, sont aussi de nature à favoriser le dialogue et à envoyer un signal fort aux employés sur l'engagement réel des dirigeants et l'importance de l'environnement dans leur stratégie. Ces contacts directs et réguliers pourront rendre le management environnemental participatif et avoir un impact positif sur la stratégie et la performance de l'entreprise, mais à condition que les employés puissent clairement s'exprimer et remettre en cause une décision sans craindre des représailles.

Gestion, Revue internationale de gestion - Vol. $36 N^{\circ} 3,2011-A . R E N A U D$ 


\section{Structurer ou institutionnaliser la préoccupation pour l'environnement en mandatant des responsables}

Pour favoriser des échanges transversaux, il est indiqué de construire des passerelles entre les fonctions et les sites géographiques de l'entreprise. Le réseau vert constitue un moyen de rassembler tous les acteurs environnementaux et de coordonner leurs actions autour d'une stratégie environnementale. Pour ce faire, ce réseau vert doit organiser périodiquement des réunions sur les préoccupations et les occasions en matière d'environnement. Les responsables et les experts environnementaux de tous les emplacements de l'entreprise pourront y débattre des problèmes courants, définir des axes de progrès pour améliorer la performance environnementale et diffuser les bonnes pratiques dans toute l'entreprise. Ces débats peuvent s'organiser de diverses manières: dans le cadre de réunions formelles, de forums de discussion, de visioconférences, de voyages organisés, etc. Dans ce dernier cas, le fait d'organiser des séminaires de deux ou trois jours, avec des séances de remue-méninges sur des thématiques environnementales précises, peut engendrer de nouvelles idées et renforcer la cohésion sociale.

Le réseau vert permet aussi de mobiliser durablement les employés qui ne sont pas spécialistes des questions environnementales. En effet, la structure décentralisée de ce réseau facilite l'engagement des employés à tous les niveaux de l'organisation. Elle permet de consulter quotidiennement les employés, de discuter avec eux des bonnes et des mauvaises pratiques et de les associer aux décisions et aux actions environnementales qui les concernent plus directement. Grâce au réseau vert, l'environnement naturel devient un facteur de mobilisation et d'apprentissage organisationnels, qui peut favoriser l'émergence de nouvelles initiatives stratégiques. Mais l'efficacité de ce réseau repose sur la liberté d'expression ; l'écoute et le respect mutuel sont à la base d'une discussion constructive.

\section{Encourager la participation des parties prenantes externes}

Dialoguer avec les parties prenantes externes est aujourd'hui indispensable, parce que la performance des entreprises est soumise à leurs attentes et que ces acteurs externes exigent de participer à la prise de 
décision des dirigeants. Pour répondre à cette exigence, plusieurs actions peuvent être conduites par les dirigeants tant au niveau national qu'au niveau local.

Par exemple, les dirigeants peuvent créer un comité de concertation au niveau national pour débattre avec leurs parties prenantes externes au sujet, par exemple, de grands projets d'investissement ou des impacts d'un projet de loi. Certaines problématiques environnementales pouvant être spécifiques d'un lieu de production, un comité régional ou local doit être mis en place pour organiser régulièrement des rencontres en face-face avec les parties prenantes externes, traiter de la performance locale et répondre de manière précise et individuelle à leurs préoccupations. Les comptes rendus de ces réunions doivent être publiés sur le site Web ou l'intranet de l'entreprise afin de favoriser la transparence sur les pratiques environnementales et l'accessibilité de ces informations au plus grand nombre.

En dehors des réunions formelles, les dirigeants peuvent aménager dans leurs locaux un espace d'accueil et de rencontre pour les parties prenantes externes, à l'instar des «maisons de l'environnement». Ils peuvent aussi mettre en place un espace de discussion virtuel (par exemple, un forum dédié aux parties prenantes) avec la participation programmée et systématique des acteurs environnementaux ou de dirigeants opérationnels de l'entreprise. Comme le montrent les organisations proactives étudiées, l'organisation de conférences-débats, de groupes de travail pour discuter de projets d'investissement, de journées portes ouvertes ou de visites d'entreprise peut aussi favoriser la participation des parties prenantes.

Au cours du processus de consultation et de dialogue avec les parties prenantes externes, les dirigeants doivent instaurer un climat de confiance et éviter un double discours sur les pratiques et la performance environnementale de leur entreprise. Les conséquences pouvant être désastreuses pour la réputation et la légitimité sociale de l'entreprise, la transparence doit être l'un des moteurs de ces échanges. En cas d'intérêts divergents entre les parties prenantes externes, les dirigeants agiront à titre d'arbitres. Gestion, Revue internationale de gestion - Vol. $36 N^{\circ} 3,2011-A$. RENAUD 
Lorsqu'une proposition d'une partie prenante est privilégiée par rapport à une autre, il est nécessaire d'expliquer clairement les raisons de ce choix aux autres parties prenantes afin qu'elles ne se sentent pas exclues.

Enfin, les parties prenantes doivent participer de manière plus opérationnelle et ne pas se limiter à observer le processus de management environnemental. Par exemple, elles peuvent participer ponctuellement aux audits internes croisés et aux revues de direction environnementales. De cette façon, leur contribution deviendra effective et le management environnemental sera véritablement perçu comme une responsabilité partagée. En d'autres termes, l'objectif est de faire évoluer la «responsabilité de l'entreprise» vers une «responsabilité de l'entreprise et de ses parties prenantes» engagées dans le management environnemental.

\section{Références}

Andriof, J., Waddock, S. (2002), «Unfolding stakeholder engagement», dans Andriof, J., Waddock, S., Husted, B., Rahman S.S. (dir.), Unfolding Stakeholder Thinking: Theory, Responsibility, and Engagement, Greenleaf, p. 19-42.

Aragon-Correa, J.A., Rubio-Lopez, E.A. (2007), «Proactive corporate environmental strategies: Myths and misunderstandings», Long Range Planning, vol. 4, $\mathrm{n}^{\circ} 3$, p. 357-381.

Baret, P. (2009), «Quatre temps pour implémenter une stratégie environnementale», Management \& Avenir, $\mathrm{n}^{\circ} 29$, p. 242-257.

Boiral, O. (2000a), «Vers une gestion environnementale des entreprises?», Revue Française de Gestion, vol. 127, p. 4-18.

Boiral, O. (2000b), «Les démarches participatives à l'épreuve de la gestion environnementale», Gestion 2000 , vol. $17, \mathrm{n}^{\circ} 4$, p. $37-51$.

Boiral, O. (2006), «L'environnement en management et le management environnemental : enjeux et perspectives d'avenir», dans Aktouf, O., Boiral, O., Mehran, E., Saives, A.-L. (dir.), Le management entre tradition et renouvellement ( $4^{\mathrm{e}}$ éd.), Gaëtan morin éditeur, p. 419-450.

Boiral, O. (2007a), Environnement et gestion : de la prévention à la mobilisation, Les Presses de l'Université Laval.

Gestion, Revue internationale de gestion - Vol. $36 N^{\circ} 3,2011-$ A. RENAUD 
Boiral, O. (2007b), «Corporate greening through ISO 14001: A rational myth?», Organization Science, vol. $18, \mathrm{n}^{\circ} 1$, p. $127-146$.

Bonnafous-Boucher, M., Pesqueux, Y. (2006), Décider avec les parties prenantes, La Découverte.

Capron, M., Quairel-Lanoizelée, F. (2007), La responsabilité sociale d'entreprise, La Découverte.

Carroll, A.B., Näsi, J. (1997), «Understanding stakeholder thinking: Themes from a finnish conference», Business Ethics, vol. 6, $\mathrm{n}^{\circ}$ 1, p. 46-51.

Dohou-Renaud, A. (2009), «Le rôle des outils de mesure de la performance environnementale : le cas des audits et indicateurs environnementaux dans dix entreprises françaises certifiées ISO 14001», Management \& Avenir, $\mathrm{n}^{\circ} 29$, p. 344-362.

Freeman, R.E. (1984), Strategic Management: a Stakeholder Approach, Pitman.

Gendron, C. (2004), La gestion environnementale et la norme ISO 14001, Les Presses Universitaires de Montréal.

ISO 14001(2004), Systèmes de management environnemental - Exigences et lignes dirctrices pour son utilisation, AFNOR.

Martinet, A., Reynaud, E. (2004), Stratégies d'entreprise et écologie, Economica.

Mitchell. R.K., Agle, B.R., Wood, D.J. (1997), «Toward a theory of stakeholderidentification and salience: Defining the principle of who and what really counts», Academy of Management Review, vol. 22, n 4, p. 853-886.

Nash, J., Ehrenfeld, J. (2001), «Factors that shape EMS outcomes in firms», dans Coglianese, C., Nash, J. (dir.), Regulating From the Inside: Can Environmental Management Systems Achive Policy Goals?, Resources for the future.

Philippe, D. (2006), «La communication environnementale organisationnelle comme stratégie de légitimation», Management international, vol. 10, n 3, p. 49-66.

Quairel-Lanoizelée, F. (2009), «Reporting "développement durable"», dans Colasse, B. (dir.), Encyclopédie de Comptabilité, Contrôle de gestion et Audit, Economica, p. 1241-1254.

Reverdy, T. (2005), «Les normes environnementales en entreprise : la trajectoire mouvementée d'une mode managériale», Sociologies Pratiques, ${ }^{\circ}$ 10, p. 97-119.

Sobczak, A., Berthoin-Antal, A. (2010), «Nouvelles perspectives sur l'engagement des parties prenantes : enjeux, acteurs, recherches», Management et Avenir, vol. 33, p. 117-126.

Suchman, M.C. (1995), «Managing legitimacy: Strategic and institutional approaches», Academy of Management Review, vol. 20, n 3, p. 571-610.

Gestion, Revue internationale de gestion - Vol. $36 N^{\circ} 3,2011-A$. RENAUD 


\section{Notes}

${ }^{1}$ Nous remercions les évaluateurs pour les remarques et les suggestions qui ont permis d'améliorer cet article.

${ }^{2}$ Capron et Quairel-Lanoizelée (2007 : 91), Qauirel-Lanoizelée (2009 : 1241).

${ }^{3}$ Gendron (2004 : 59).

${ }_{5}^{4}$ ISO 14001(2004).

${ }_{5}$ The ISO Survey of Certifications 2009, www.iso.org/iso/survey2009.pdf; www.iso.org/iso/iso_catalogue/management_standards/certification/the_iso_survey.htm.

${ }^{6}$ En France, la notion de «management environnemental» est préférée à celle de «gestion environnementale», car c'est celle qui s'est imposée dans les pratiques organisationnelles.

${ }^{7}$ Voir, par exemple, les travaux de Boiral (2000a, 2000b, 2006, 2007a, 2007b), Nash et Ehrenfeld (2001), Philippe (2006), Reverdy (2005), Dohou-Renaud (2009), Baret (2009).

${ }^{8}$ Suchman (1995).

${ }^{9}$ Boiral (2000b; 2006 : 444).

${ }^{10}$ Nash et Ehrenfeld (2001).

${ }^{11}$ Boiral (2006 : 444).

${ }^{12}$ Le concept de «parties prenantes» (stakeholders) désigne «des individus ou des groupes d'individus qui peuvent influencer la réalisation des objectifs de l'organisation ou être influencés par celle-ci» (Freeman, 1984 ; traduction libre).

${ }^{13}$ Bonnafous-Boucher et Pesqueux (2006).

${ }^{14}$ Baret (2009).

${ }^{15}$ Gendron (2004).

${ }^{16}$ Andriof et Waddock (2002).

${ }^{17}$ Gendron (2004), Martinet et Reynaud (2004), Capron et Quairel-Lanoizelée (2007), Aragon-Correa et RubioLopez (2007).

${ }^{18}$ Cette typologie rappelle celle de Carroll et Näsi (1997) qui opposent les parties prenantes internes (propriétaires, dirigeants, employés) aux parties prenantes externes (concurrents, consommateurs, gouvernements, groupes de pression, médias, communauté et environnement naturel).

${ }^{19}$ Gendron (2004), Boiral (2006).

${ }^{20}$ Dans les petites et moyennes entreprises de notre échantillon, la fonction «environnement» est également décentralisée même si elle se réduit à peu d'individus (un responsable de l'environnement rattaché à la direction de l'entreprise, un animateur de l'environnement chargé du système de management environnemental).

${ }^{21}$ Les «maisons d'accueil de l'environnement» ou «maisons de l'environnement» sont des lieux de rencontres qui ont pour mission de répondre aux demandes d'information du public et d'organiser des expositions, des débats et des manifestations autour des questions écologiques. 